\title{
FLT-PET may not be a reliable indicator of therapeutic response in p53-null malignancy
}

\author{
SHARYN I. KATZ ${ }^{3 *}$, LANLAN ZHOU ${ }^{1,5^{*}}$, THOMAS A. FERRARA ${ }^{3}$, WENGE WANG $^{6}$, \\ PATRICK A. MAYES ${ }^{7}$, CHARLES D. SMITH ${ }^{4}$ and WAFIK S. EL-DEIRY ${ }^{1,2}$
}

\begin{abstract}
${ }^{1}$ Laboratory of Translational Oncology and Experimental Cancer Therapeutics, Department of Medicine (Hematology/Oncology), Penn State College of Medicine, Penn State Hershey Cancer Institute, Penn State Hershey Medical Center, Hershey, PA $17033 ;{ }^{2}$ The American Cancer Society, Atlanta, GA 30329; ${ }^{3}$ Department of Radiology,

Institute for the Translational Medicine and Therapeutics, Abramson Cancer Center, University of Pennsylvania

School of Medicine; ${ }^{4}$ Medical University of South Carolina, Charleston, SC; ${ }^{5}$ Department of Bioengineering, School of Engineering and Applied Science, University of Pennsylvania; ${ }^{6}$ Department of Hematology/Oncology, Children's Hospital of Philadelphia, Philadelphia, PA; ${ }^{7}$ Department of Medicine, Mount Vernon Hospital, Mount Vernon, NY, USA
\end{abstract}

Received February 10, 2011; Accepted March 4, 2011

DOI: 10.3892/ijo.2011.1019

\begin{abstract}
FDG $\left({ }^{18} \mathrm{~F}\right.$-deoxy-glucose) is the current gold standard for PET imaging. FLT (3'-deoxy-3'-( ${ }^{18}$ F-fluorothymidine), a PET imaging marker of proliferation, has been proposed as an alternative to FDG for the assessment of therapeutic response. We examined the therapeutic predictive value of FLT-PET and FDG-PET using CALU-6, a human, p53-null, non-small cell lung cancer cell line with comparison of combined targeted therapy, TRAIL and sorafenib, versus combined conventional chemotherapy, docetaxel and cisplatin. CALU-6 tumor-bearing $n u / n u$ mice $(\mathrm{n}=46)$ were evaluated in 3 therapeutic trials measuring FLT and FDG prediction of tumor response at $72 \mathrm{~h}$ following initiation of daily combination therapy with targeted agents, TRAIL (200 $\mu$ g i.v.) and sorafenib ( $30 \mathrm{mg} / \mathrm{kg}$ i.p.) and compared to conventional chemotherapeutics cisplatin ( $3 \mathrm{mg} /$ $\mathrm{kg}$ i.p.) and docetaxel (7.5 $\mathrm{mg} / \mathrm{kg}$ i.p.). PET imaging response was compared to morphological and histological indicators of therapeutic response, including decreased vascularity (in vivo AngioSense imaging and anti-CD31 staining), slowed tumor growth (caliper measurements), decreased cellular proliferation (Ki-67 staining) and increased apoptosis (TUNEL staining). Decreases in tumor accumulation of FLT $\left(\mathrm{FLT}_{\mathrm{MAX}}-30 \%\right.$, $\mathrm{p}=0.03$ ) at $72 \mathrm{~h}$ post treatment were observed in response to TRAIL and sorafenib combination therapy resulting in smaller,
\end{abstract}

Correspondence to: Dr Sharyn I. Katz, Department of Radiology, University of Pennsylvania School of Medicine, 1 Silverstein Building, 3400 Spruce Street, Philadelphia, PA 19104, USA

E-mail: sharyn.katz@uphs.upenn.edu

${ }^{*}$ Contributed equally

Key words: FLT, PET, p53, non-small cell lung cancer less vascular, more apoptotic tumors. No similar reduction in tumor accumulation of FLT $\left(\mathrm{FLT}_{\mathrm{MAX}}-2 \%, \mathrm{p}=0.67\right)$ was observed $72 \mathrm{~h}$ following initiation of cisplatin and docetaxel combination therapy, despite histological and morphological evidence of drug response. In contrast, tumor imaging with FDG did demonstrate a decrease in accumulation in both treatment groups, $-21 \%(\mathrm{p}=0.30)$ in response to cisplatin/ docetaxel and $-8 \%(\mathrm{p}=0.59)$ in response to TRAIL/sorafenib, but did not reach statistical significance. FLT, but not FDG, is predictive of therapeutic response to the targeted regimen TRAIL/sorafenib. However, FLT-PET may not predict therapeutic response to DNA damaging agents in p53-null tumors, likely due to loss of cell cycle control of thymidine kinase 1 (TK1). Thus, tumor imaging response by FLT may be limited in human tumors without functional p53.

\section{Introduction}

Lung cancer, which is predominantly p53 insufficient (1-3), is often diagnosed at an advanced stage with less than 1-year survival. A major barrier to improving these survival statistics stems from our current system of reliance on large changes in tumor size to assess therapeutic response, with typical posttherapeutic CT scan occurring months after therapy initiation (4). Recently, PET imaging radiotracers of metabolism have been explored as early markers of therapeutic response in malignancy. FDG, a PET radiotracer of glucose metabolism, is currently the clinical radiotracer of choice for oncologic PET applications.

While FDG-PET is highly sensitive and can serve as a marker of therapeutic response, specificity suffers and is particularly limited in certain therapeutic settings such as following radiation therapy, surgery, infection and talc pleurodeisis and in certain anatomic locations including the brain, mediastinum, heart and liver. FLT $\left({ }^{18} \mathrm{~F}\right.$-labelled thymidine), is a PET (positron emission tomography) imaging thymidine analog radiotracer, and well documented surrogate marker of 
cellular proliferation, which has shown promise as an early therapeutic response indicator. FLT-PET tumor response to therapy, correlating ultimately with morphological tumor response, has been demonstrated as early as $24-48 \mathrm{~h}$ following chemotherapy initiation, both in animal models (5-14) and clinical trials (15-25).

In order to implement FLT into clinical practice, triaging chemotherapies early after induction, the variables impacting FLT accumulation in tumors must be fully understood. FLT enters the cell using cell membrane-associated nucleoside transporters then is retained in the cell after phosphorylation by thymidine kinase 1 (TK1), a tightly regulated enzyme and rate-limiting step of the DNA salvage pathway, vital for maintaining tumor thymidine levels. Since thymidine is not a component of RNA, tumor accumulation of FLT is a reflection of DNA synthetic rate only and correlates exceptionally well with TK1 activity and percentage of cells in S phase of the cell cycle. TK1 is virtually absent in non-dividing cells (26).

In this report, we examine changes in tumor accumulation of FLT and FDG following therapy in a xenograft mouse model of p53-null human non-small cell lung cancer (NSCLC) using CALU-6. PET imaging tumor response is evaluated after $72 \mathrm{~h}$ of therapy with either a conventional,DNA-damaging chemotherapy regimen or targeted regimen. We chose CALU-6, a p53-null human NSCLC line which has been previously characterized as sensitive to monotherapy with conventional therapies cisplatin (27-31), a DNA damaging agent, and docetaxel $(32,33)$, an anti-tubulin agent, in order to examine the response of FLT to traditional chemotherapeutics. Cisplatin and docetaxel combination therapy is a currently accepted conventional first-line therapy for NSCLC.

CALU-6 cells are also very sensitive to combination therapy with the targeted agents TRAIL (TNF-related apoptosis inducing ligand), a naturally occurring death-receptor agonist, and sorafenib, a multi-targeted tyrosine kinase inhibitor (34). Both death receptor agonists and sorafenib have demonstrated evidence of efficacy in NSCLC and are currently in clinical trials (35-39). Examination of p53-null tumor FLT response to the targeted therapies TRAIL and sorafenib provides an opportunity to study FLT response in a system known to be therapeutically effective in vitro and not reliant on DNA-damage as the principle mechanism of action.

\section{Materials and methods}

Cell lines and cell culture. CALU-6, a p53-null human non-small cell lung cancer cell line was newly purchased from American Type Culture Collection (ATCC, Manassas, VA).

Cells were maintained in a humidified sterile environment with $5 \% \mathrm{CO}_{2}, 37^{\circ} \mathrm{C}$ RPMI-medium supplemented with $10 \%$ heat-inactivated fetal calf serum with $1 \%$ penicillin and streptomycin (CM). Passage of cell lines was performed at 1:5 dilution after detachment using sterile $0.05 \%$ trypsin-EDTA solution.

Chemotherapeutics. His-tagged-TRAIL was synthesized via plasmid expression as previously described (40) and purification and stored as a $1 \mathrm{mg} / \mathrm{ml}$ stock in $\mathrm{dPBS}$ at $-20^{\circ} \mathrm{C}$. Sorafenib was synthesized in powder, free base form for use in our laboratory as previously described (41). Sorafenib was dissolved in $100 \%$
DMSO and stored at $-20^{\circ} \mathrm{C}$ as a concentrated stock for in vitro studies. For in vivo studies, sorafenib was dissolved in a Cremaphor EL solution (Sigma-Aldrich, St. Louis, MO), made freshly for each daily dose. Cisplatin powder was purchased from (Sigma-Aldrich) and prepared freshly in sterile dPBS prior to each injection. Docetaxel $(10 \mathrm{mg} / \mathrm{ml}$ solution) was obtained from the University of Pennsylvania Abramson Cancer Center pharmacy then diluted in the 13\% ETOH vehicle solution.

In vitro cell death assays. Cells were harvested from culture with $0.05 \%$ trypsin-EDTA, washed in CM, plated at $0.6 \times 10^{6} \%$ well in a sterile 6-well plate and incubated at $37^{\circ} \mathrm{C}, 5 \% \mathrm{CO}_{2}$ for 18-24 $\mathrm{h}$ to allow attachment of cells to the wells. Sorafenib (diluted in CM from $32 \mathrm{mM}$ stock in DMSO) was then added to the appropriate wells for a total of $4 \mathrm{ml} \mathrm{CM}$ and incubated for 16-22 h. Cells treated with TRAIL (his-TRAIL, diluted in CM) were incubated with TRAIL for $4 \mathrm{~h}$ with or without pre-incubation with sorafenib, and added to the appropriate wells at either 50 or $100 \mathrm{ng} / \mathrm{ml}$.

For wells not containing sorafenib, culture medium was treated with $0.1 \%$ DMSO to control for the possible effects of DMSO on cell viability. After incubation with treatment, cells were washed with cold sterile dPBS, fixed (10\% methanol/10\% acetic acid solution) for $10 \mathrm{~min}$, stained with Coomassie blue for $3 \mathrm{~min}$, then rinsed with distilled water and allowed to air-dry.

Propidium iodide FACS. After incubations with chemotherapies in a $60-\mathrm{mm}$ tissue culture dish, supernatants and adherent cells were removed using $0.5 \%$ trypsin-EDTA, washed with cold dPBS and cell pellets resuspended in a $75 \% \mathrm{ETOH}$ solution $\left(4^{\circ} \mathrm{C}\right)$ for a period of overnight to 1 month at $4^{\circ} \mathrm{C}$. On the day prior to FACS analysis, cells were washed with cold dPBS, permeabilized with $0.5 \mathrm{M}$ phosphocitrate acid solution, then resuspended in a propidium iodide solution (RNAse/PI in dPBS) and allowed to incubate overnight at $4^{\circ} \mathrm{C}$. The cells were then sorted by FACS the next day and the subG ${ }_{1}$ fraction measured.

Xenograft formation. Prior to the use of animal models, protocol approval was obtained by the Institutional Animal Care and Use Committee (IACUC) at the University of Pennsylvania. Two-month old female $n u / n u$ mice (Crl:NUFoxn1nu, Charles River Laboratory, Wilmington, MA) were injected subcutaneously in each flank with $2 \times 10^{6}$ CALU-6 cells suspended in a sterile, endotoxin-free $50 \%$ Matrigel solution. After therapy/imaging completion, mice were euthanized with ketamine i.p. followed by cervical dislocation.

In vivo therapeutic trials. Following xenograft formation, FLT-PET or FDG-PET was performed prior to initiation of chemotherapy (Fig. 1). Sorafenib was administered at a dose of $30 \mathrm{mg} / \mathrm{kg}$ diluted in a Cremaphor EL/13\% ETOH vehicle solution and injected i.p. daily. TRAIL was diluted in sterile PBS and injected by tail vein at $200 \mu \mathrm{g}$, daily. Cisplatin was diluted in sterile PBS and injected i.p. at $3 \mathrm{mg} / \mathrm{kg}$ daily. Docetaxel was obtained freshly diluted from the Abramson Cancer Center pharmacy in a $13 \%$ ETOH vehicle solution and injected i.p. at $7.5 \mathrm{mg} / \mathrm{kg}$ daily. Both cisplatin and docetaxel were dosed weekly. 


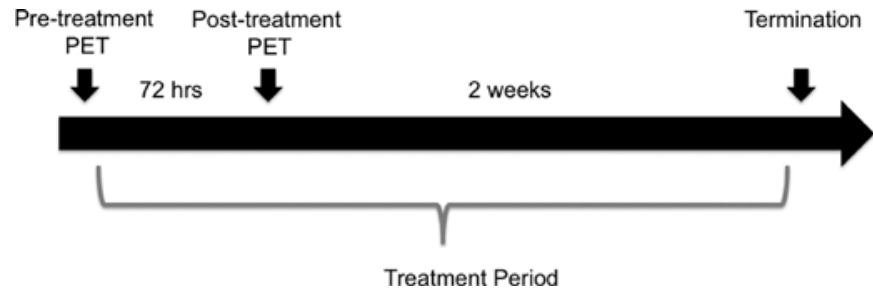

Figure 1. Imaging algorithm for PET imaging. Immediately prior to initiating chemotherapy, a baseline pre-therapy FDG-PET or FLT-PET was acquired At $72 \mathrm{~h}$ following initiation of therapy, a post-treatment PET was performed Therapy was continued for $\sim 2$ weeks, followed by euthanasia and histological examination of tumor tissues.

In vivo trials were conducted in order to assess the dynamics of FLT-PET uptake in CALU- 6 tumor-bearing xenografts treated with TRAIL and sorafenib, for an average therapeutic period of 15 days. Either FLT-PET or FDG-PET was performed at baseline and $72 \mathrm{~h}$ after therapy initiation. At the completion of the therapeutic period, mice were euthanized and tumors excised and preserved for histology in a $4 \%$ paraformaldehyde solution. Tissues were then paraffin-embedded, sectioned and stained with hematoxylin and eosin, antibody against Ki-67 nuclear antigen (Dako, Carpinteria, CA), anti-CD31 (Dako), and TUNEL (Roche Diagnostics Corp., Indianapolis, IN). If tumors became too large to treat, intravenous access became limited, or mice demonstrated signs of distress, mice were sacrificed immediately as per IACUC regulations. Out of a total of 46 xenografted mice, only 1 died prior to completion of the trial, this mouse was in the treatment group during the pilot trial of TRAIL and sorafenib therapy.

A pilot trial of 8 CALU-6 tumor-bearing mice receiving daily TRAIL/sorafenib therapy $(n=4)$ or untreated $(n=4)$ was conducted in order to assess success of the in vivo anti-angiogenic therapy with the TRAIL/sorafenib regimen. At the completion of the therapeutic period, and prior to sacrifice, tumors were imaged with the optical imaging agent AngioSense (VisEn Medical, Inc., Bedford, MA) as per vendor specifications. Once tumor response to sorafenib/TRAIL therapy was established in vivo, a cohort of 25 tumor-bearing mice were allocated to one of the following groups: cisplatin/docetaxel (n=8); TRAIL/ sorafenib $(n=8)$; untreated control $(n=5)$; or vehicle control $(\mathrm{n}=4)$. In addition to following therapeutic response by in vivo tumor imaging and ex vivo histology, tumors were also followed by caliper volume measurements using the modified

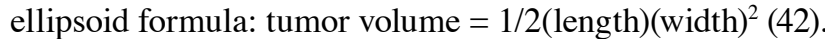

Finally, to assess whether FLT-PET or FDG-PET could predict treatment failure, despite inherent CALU-6 sensitivity to therapy, tumors were allowed to reach $2-3$ times the size of optimal treatment size $\left(330-350 \mathrm{~mm}^{3}\right.$ rather than $\left.100-150 \mathrm{~mm}^{3}\right)$; we have observed in our laboratory that growth of tumors to this size significantly hinders therapeutic efficacy in xenograft models of malignancy. Tumor-bearing mice were then assigned to either treatment with TRAIL/sorafenib $(n=7)$ or no treatment $(n=6)$ groups. One of the mice from each the untreated and treated groups was dropped from the data calculations due to poor tumor growth.

PET imaging and image interpretation. FLT and FDG radiotracers were synthesized in the PET Cyclotron Facility at the University of Pennsylvania. After anesthesia with inhaled isofluorane in $\mathrm{O}_{2}$ (3\% induction, $1.5 \%$ maintenance), mice were injected with $200-250 \mu \mathrm{Ci}$ of FLT or FDG then allowed to recover from the anesthesia during the 60-min uptake time allowed for radiotracer accumulation. At $60 \mathrm{~min}$ post-injection, mice were anesthetized and imaged for a $15-\mathrm{min}$ static acquisition on the small animal PET scanner (A-PET, built in collaboration with Philips Medical Systems) located at the University of Pennsylvania Small Animal Imaging Facility. This system has very high spatial resolution $(\sim 2 \mathrm{~mm})$, operates exclusively as a fully 3D instrument with very high sensitivity and large field-of-view of $12 \mathrm{~cm}$ in both axial and transverse directions.

Images were reconstructed with the manufacturer software and tumor volumes of FLT quantitated with the freely available Amide 3D software (Medical Image Data Examiner (Amide), LG Software Innovations), which allows for multiplanar analysis of the tumor volume. A ROI (region of interest) was traced around the tumor volume creating a 3D ROI that could be carefully sculpted to the 3D tumor perimeter and visually inspected in the axial, coronal and sagittal planes. Once 3D tumor volume is created, the Amide software provides statistical data analysis including ROI volume $\left(\mathrm{mm}^{3}\right)$, mean counts/pixel, max PET counts/pixel and standard deviation of tumor counts/ pixel. PET values generated by the A-PET machine are absolute pixel counts. Absolute tumor counts were normalized to flank muscle (tumor ROI pixel value/thigh muscle ROI pixel value). This normalization provides a correction factor for subtle differences in PET tumor uptake time and dose.

Optical imaging. At $48 \mathrm{~h}$ prior to sacrifice, each mouse was anesthetized with i.p. ketamine and injected with $100 \mu \mathrm{g}$ of AngioSense IV (VisEn Medical, Inc.) by tail vein and a time course of imaging tumor imaging with AngioSense (preinjection, $20 \mathrm{~min}, 6,24$ and $48 \mathrm{~h}$ ) was acquired on the Maestro (CRI, Woburn MA), with appropriate excitation and absorbance filters. The AngioSense signal was acquired as an image cube data file, then dye signal was unmixed from background autofluorescence using the CRI post-processing software. Immediately after euthanasia, the tumors were excised and AngioSense signal measured directly from the tumor on the Xenogen IVIS system using the ICG channel. AngioSense imaging was performed for the pilot trial of TRAIL/sorafenib therapy to evaluate therapeutic response.

Histological analysis. After euthanasia, tumors were promptly excised, weighed, immersed in a $4 \%$ paraformaldehyde solution and allowed to incubate overnight at $4{ }^{\circ} \mathrm{C}$. The tissues were then removed and placed in a solution of $70 \%$ ethanol for $24 \mathrm{~h}$ to 2 weeks at $4^{\circ} \mathrm{C}$. Tissues were then paraffin-embedded, sectioned into $5 \mu \mathrm{m}$ slices and mounted onto slides by the GI Morphology Core, University of Pennsylvania. Immunohistochemistry was performed at the Children's Hospital of Philadelphia Research Institute, Pathology Core. TUNEL staining was performed using TUNEL enzyme and TUNEL label (Roche Boehringer-Mannheim, Indianapolis, IN).

TUNEL stained tissues were viewed (x20 magnification) under a Nikon E600 microscope (Nikon, Japan), equipped with fluorescent optics, and 5 randomly acquired $22 \mathrm{~mm}$ fieldof-view (FOV) images were obtained for each slide. Two 


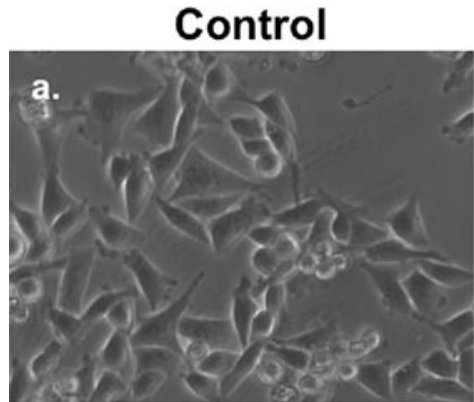

Sorafenib

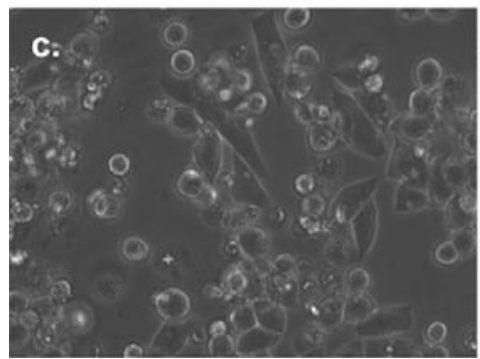

TRAIL

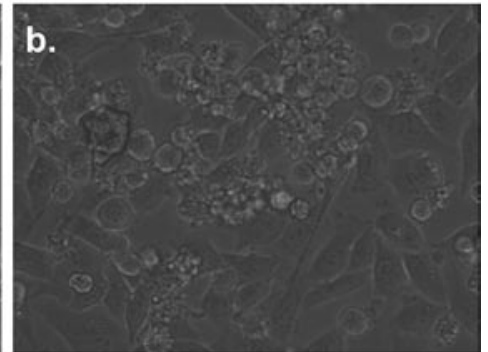

Sorafenib + TRAIL

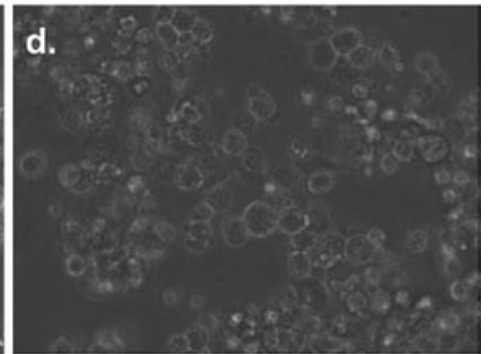

Figure 2. TRAIL and sorafenib therapy induced robust apoptosis in CALU-6, in vitro. Brightfield microscopy images of CALU-6 cultures reveals normal angular, smooth, adherent cells in the untreated control (a) with little change after the addition of TRAIL alone. Modest sensitivity to sorafenib (c) is manifested by smaller, irregular, detached floating apoptotic cells. Synergistic cell killing results from of combination therapy with TRAIL and sorafenib.

images were captured for each FOV optimized for each spectrum for TUNEL staining (absorption ${ }_{\mathrm{MAX}} 365 \mathrm{~nm}$, emission $_{\text {MAX }} 460 \mathrm{~nm}$; green filter) and DAPI counterstaining (absorption $_{\text {MAX }} 358 \mathrm{~nm}$, emission MAX $461 \mathrm{~nm}$; blue/cyan filter). Nuclear TUNEL and DAPI staining positivity was then quantitated using Image $\mathbf{J}$ software (Image Processing and Analysis in Java, National Institute of Health, Bethesda, MD). The $\%$ TUNEL-positive nuclei was normalized using $\%$ positive DAPI nuclear staining for each FOV. The mean normalized \% TUNEL staining value of the 5 acquisitions for each tumor was then calculated. Tissues stained with anti-Ki-67 (Dako, Carpinteria, CA) were developed with horseradish-peroxidase linked antibodies and the entire slide for each tumor was scanned into digital format. The $\%$ positive nuclear staining was calculated using the semi-automated Aperio scanscope software.

Statistical analysis. Quantitative data were analyzed, including t-test, average, linear regression and correlation, using the EXCEL Spreadsheet Data ToolPak (Microsoft Office). For paired data sets, pre and post PET imaging data, a paired, 2-tailed t-test was performed and p-value obtained. For unpaired data sets, comparisons between treatment groups, an un-paired, 2-tailed t-test was performed. An $\alpha$ of 0.05 was employed to determine significance.

\section{Results}

In this study we sought to evaluate whether FLT-PET could be used as a predictor of therapeutic success or failure in vivo in the setting of a p53-null malignancy. The p53-null human non-small cell lung cancer (NSCLC) line CALU-6 is highly sensitive to combination therapy with sorafenib and TRAIL in vitro (34). In this study we confirmed in vitro sensitivity of CALU-6 to combination therapy with TRAIL and sorafenib (Fig. 2) then further demonstrated this sensitivity in vivo, for ability to induce apoptosis (Fig. 3), inhibit angiogenesis (Fig. 4) and effect growth inhibition. In vivo therapeutic response of CALU-6 to TRAIL and sorafenib therapy thus was confirmed by the observation of markedly increased apoptosis by TUNEL staining of treated tumor tissues (Fig. 3), decreased caliper measured tumor volumes, decreased tumor accumulation of AngioSense (Fig. 4), a blood pooling agent and decreased tissue vessel density by anti-CD31 staining (Fig. 4).

P53-null CALU-6 tumor decreased accumulation of FLT at $72 \mathrm{~h}$ correlates with growth inhibition in the TRAIL/sorafenib group. TRAIL/sorafenib therapeutic response of CALU-6 tumors was predicted by a decrease in tumor accumulation of FLT at $72 \mathrm{~h}$ of therapy relative to baseline. In the pilot trial $(\mathrm{n}=8)$ of TRAIL/sorafenib therapy, the tumor overall FLT MAX $_{\text {had }}$ dropped to $-17 \%$, corresponding to actual tumor regression with a $52 \%$ decrease in mean tumor volume observed at the end of the therapeutic period. By comparison, untreated tumors demonstrated a rise in $\mathrm{FLT}_{\mathrm{MAX}}$ of $9 \%$, corresponding to a mean change in tumor volume of $206 \%(\mathrm{p}=0.05)$.

A cohort of 25 CALU- 6 bearing mice were then allocated into the following treatment groups: targeted therapy with TRAIL/sorafenib $(n=8)$; conventional DNA-damaging therapy with cisplatin and docetaxel $(n=8)$; untreated control $(n=5)$; and vehicle-only control $(n=4)$. One-half of each treatment group was serially imaged only with FLT-PET while the other half was imaged only with FDG-PET. After $72 \mathrm{~h}$ of daily TRAIL/sorafenib combination therapy, the mean tumor FLT $_{\text {MAX }}$ dropped to $-30 \%( \pm 8 \%, \mathrm{p}=0.03)$ relative to baseline (Fig. 5) corresponding to $79 \%$ tumor growth inhibition $( \pm 27 \%$, 


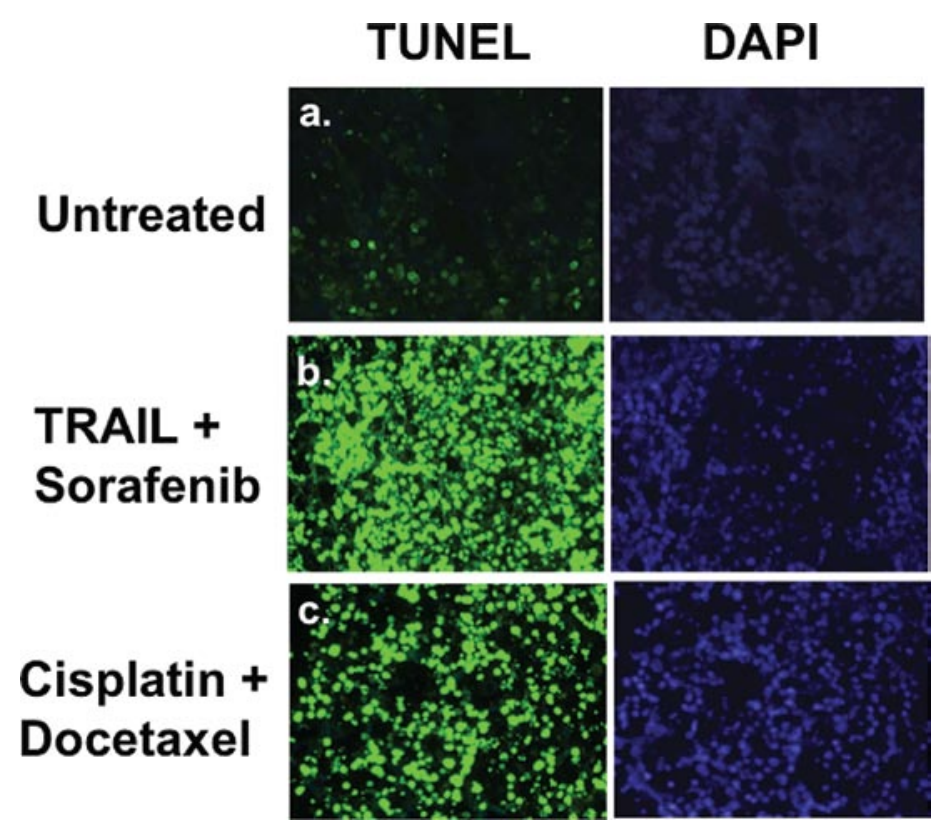

Figure 3. Both TRAIL/sorafenib and cisplatin/docetaxel therapy induced robust apoptosis in CALU-6, in vivo. TUNEL staining (green), with DAPI counterstaining (blue) of xenograft tumors excised following 2 weeks of therapy reveals robust apoptosis induced by treatment with (b) TRAIL/sorafenib and (c) cisplatin/docetaxel therapy relative to (a) untreated controls.

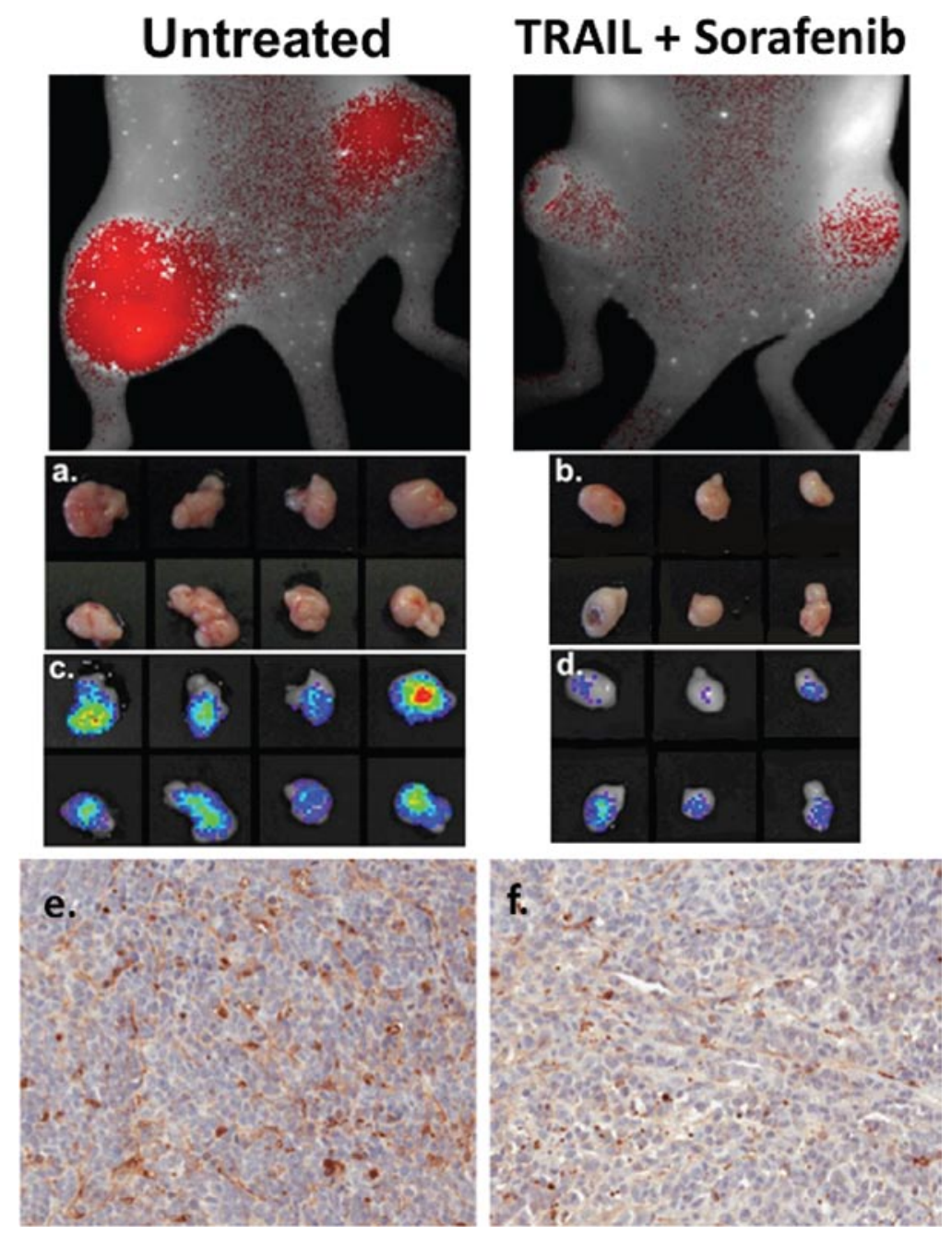

Figure 4. Treatment of CALU-6 xenografts with daily TRAIL/sorafenib results in decreased tumor vascularity. AngioSense imaging reveals marked decreased intratumoral vascularity after TRAIL/sorafenib relative to untreated controls (top row). Ex vivo, the AngioSense signal remains robust within the xenografted tumors (c, untreated; d, treated) and white light images reveal smaller, smoother, paler tumors in the TRAIL/sorafenib treated group (a) relative to untreated controls (b). These findings were confirmed with anti-CD31 IHC of xenograft tumors (e, untreated; f, TRAIL/sorafenib). 


\section{Trail/Sorafenib}

FLT-PET

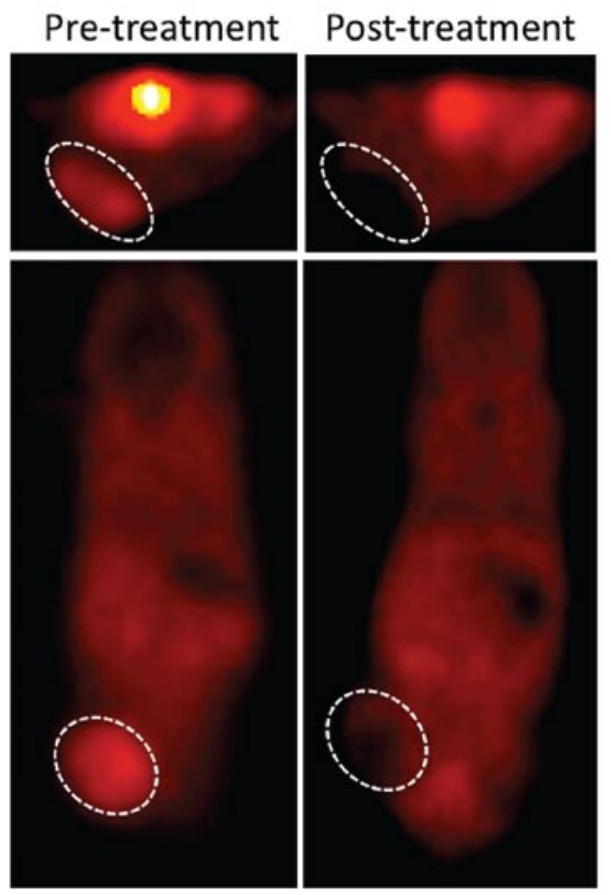

\section{FDG-PET}

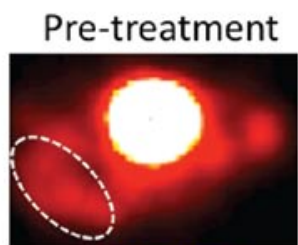

Post-treatment
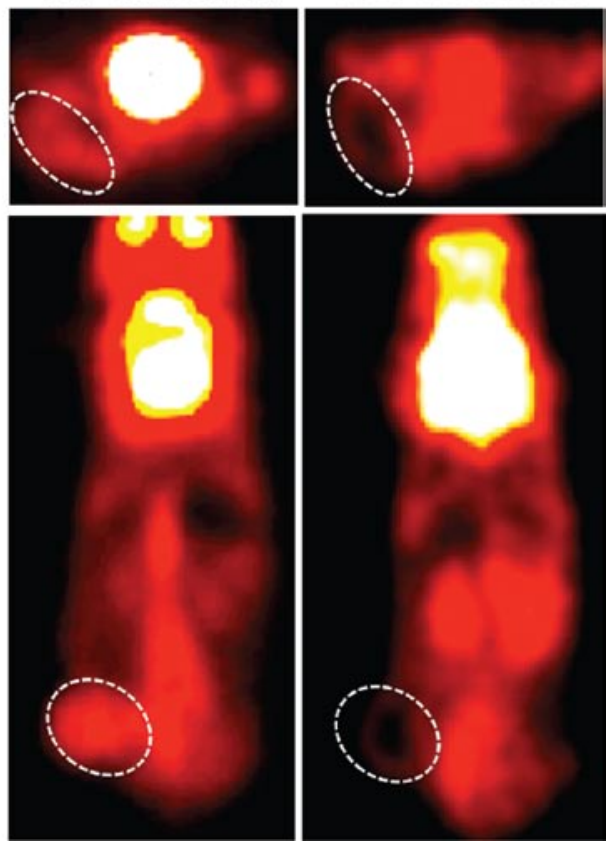

Figure 5. p53-null CALU-6 xenografts treated with TRAIL/sorafenib yielded both FLT-PET and FDG-PET imaging responses at $72 \mathrm{~h}$. FLT (left), was predictive of CALU-6 tumor response to TRAIL/sorafenib therapy as evidence by the significant drop in tumor accumulation of TRAIL/sorafenib at $72 \mathrm{~h}$. While overall, tumor accumulation of FDG accumulation also fell in response to TRAIL/sorafenib therapy, significant variability in tumor uptake blunted the significance of this response. PET images displayed are from 2 separate mice, one for each the set of FLT images and FDG images. The top row represents axial plane through the tumor with corresponding coronal view immediately below.

\section{Cisplatin/Docetaxel}

FLT-PET
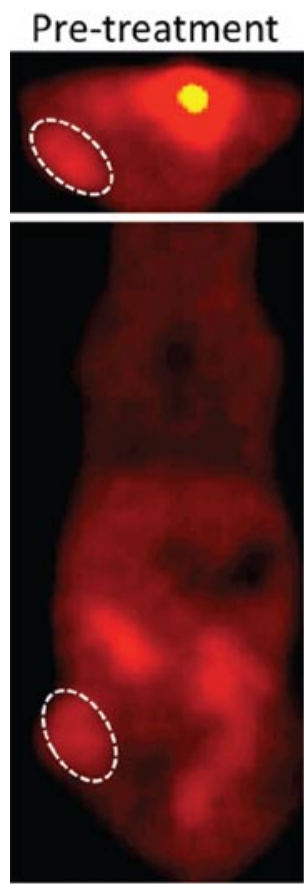

\section{Post-treatment}
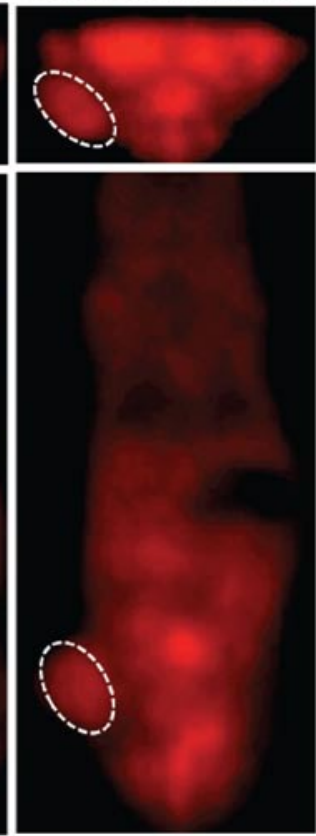

FDG-PET
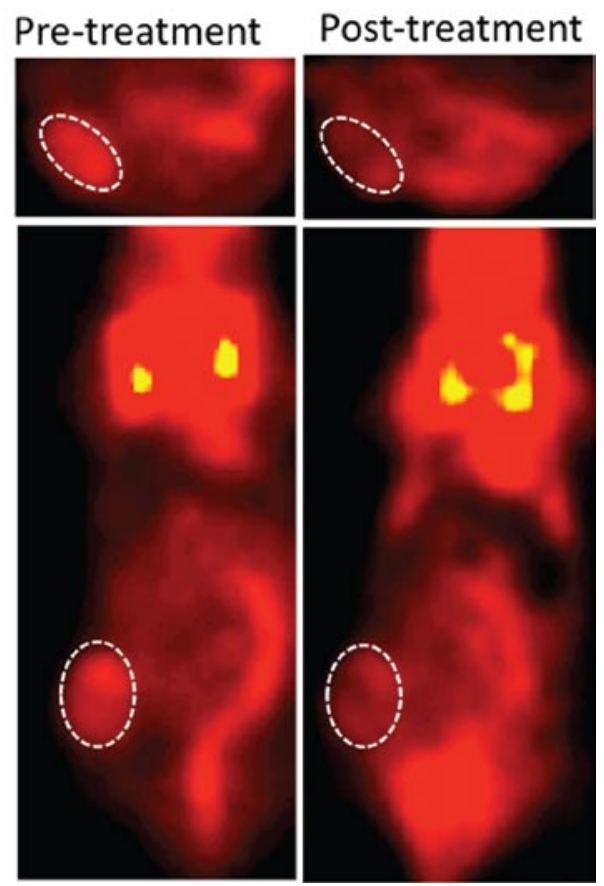

Figure 6. p53-null CALU-6 xenografts treated with cisplatin/docetaxel did not yield an FLT-PET imaging response at 72 h. CALU-6 tumors treated with DNA-damaging agents cisplatin/docetaxel did not drop accumulation of FLT in prediction of the observed treated endpoint morphological tumor response. Again, tumor accumulation of FDG dropped after cisplatin/docetaxel therapy but did not reach statistical significance due to the high variability in imaging response. PET images displayed are from 2 separate mice, one for each the set of FLT images and FDG images. The top row represents axial plane through the tumor with corresponding coronal view immediately below. 
$\mathrm{p}=0.02$ ) relative to untreated controls. Surprisingly, therapy with Cremaphor EL vehicle alone resulted in a growth inhibition of $51 \%(\mathrm{p}=0.05)$ which correlated to elevated apoptosis with subsequent TUNEL staining of excised tumor. This is a previously reported property of Cremaphor EL (43) and tempers conclusions regarding the efficacy of TRAIL/sorafenib therapy, the establishment of which is not the goal of this study. It would be useful to explore this apparent anti-tumor effect since Cremaphor EL is a commonly used vehicle for sorafenib in animal imaging.

Imaging and volume measurements correlated with histological markers of tumor response to therapy. TUNEL staining of excised tumors at end of therapy revealed increased nuclear staining for nicked DNA, indicating cellular commitment to apoptosis, in the TRAIL/sorafenib group relative to untreated controls ( $71 \%$ versus $18 \%, \mathrm{p}=1.7 \mathrm{E}-07)$. Ki-67 staining of excised tumors did not demonstrate suppression of proliferation in the TRAIL/sorafenib treatment group relative to the untreated control group ( $80 \%$ versus $80 \%$ respectively, $\mathrm{p}=0.9$ ).

P53-null CALU-6 tumor did not demonstrate an imaging response with FLT-PET response at $72 \mathrm{~h}$ despite growth inhibition in the cisplatin/docetaxel group. Combination therapy with conventional chemotherapeutics, cisplatin and docetaxel $(n=8)$ was compared to PBS vehicle $(n=2)$ and the untreated control group $(n=5)$. Cisplatin/docetaxel therapy, dosed weekly, resulted in no significant change in tumor $\mathrm{FLT}_{\mathrm{MAX}}-2 \%(\mathrm{STD} \pm 7 \%, \mathrm{p}=0.3)$ at $72 \mathrm{~h}$ following therapy initiation (Fig. 6). This lack of FLT tumor response significantly underestimated the actual mean growth inhibition of $46 \%$ (STD $\pm 30 \%, p=0.002)$. The cisplatin/docetaxel mediated growth inhibition of $46 \%$ was less robust $(p=1.19 \mathrm{E}-05)$ than that observed in the TRAIL/sorafenib group (79\%).

Cisplatin/docetaxel treated CALU-6 tumor histology demonstrated elevated proliferation post therapy. Ki-67 staining of excised CALU-6 tumors revealed slightly elevated cellular proliferation in cisplatin/docetaxel treated tissues relative to TRAIL/sorafenib (86\% versus $80 \%, \mathrm{p}=0.03$ ). TRAIL/sorafenib therapy did not appear to impact proliferation relative to untreated controls ( $80 \%$ versus $80 \%, \mathrm{p}=0.89)$. However, tissues were harvested $24 \mathrm{~h}$ after last TRAIL/sorafenib dose and 4 days after the weekly cisplatin/docetaxel dose. It is possible that cellular proliferation had begun to recover at the point of tumor excision. In addition, cisplatin/docetaxel has been previously demonstrated to transiently stimulate TK1 activity, a known response to DNA damage.

Both TRAIL/sorafenib and cisplatin/docetaxel groups demonstrated elevated apoptosis in treated CALU-6 tumors. TUNEL staining of excised cisplatin/docetaxel treated tumors revealed increased $\%$ positive cellular nuclear staining for nicked DNA, indicating commitment to programmed cell death relative to untreated controls $(40 \%$ versus $18 \%, \mathrm{p}=0.01)$ although not as robust as that induced with TRAIL/sorafenib therapy $(71 \%$ versus $18 \%, \mathrm{p}=0.0001)$. The difference in $\%$ TUNEL positivity between cisplatin/docetaxel and TRAIL/ sorafenib groups was significantly different (40\% versus $71 \%$, $\mathrm{p}=0.002)$. Interestingly, the Cremaphor EL control exhibits elevated TUNEL positivity indicating a strong pro-apoptotic characteristic of the vehicle itself $(90 \%$ versus $18 \%, \mathrm{p}=1.7 \mathrm{E}-06)$ and calling into question its utility as a chemotherapeutic vehicle. However, since testing of chemotherapeutic efficacy was not the goal of this study and the Cremaphor EL vehicle group small $(\mathrm{n}=2)$, this phenomenon merits further investigation. Interestingly, there is a report in the literature supporting the anti-tumor effects of Cremaphor EL (43).

In the setting of treatment failure, tumor $F L T_{\text {Max }}$ follows actual tumor response rather than inherent tumor cell sensitivities. In order to create a treatment failure, we allowed the CALU-6 tumors to reach a size of $330-350 \mathrm{~mm}^{3}, \sim 2-3$ times that of the usual starting tumor volume $\left(100-150 \mathrm{~mm}^{3}\right)$. In our laboratory, we have observed that when xenografted human tumors are allowed to grow to large sizes, they are more refractory to treatment, independent of inherent sensitivities (unpublished data). Using this treatment failure model, the TRAIL/sorafenib treatment group $(n=7)$ and untreated control $(n=6)$ both demonstrated tumor growth with the exception of one mouse in the untreated group that did not grow. The data from this one mouse was excluded from the results. Interestingly, tumor uptake of FLT did not decrease in response to TRAIL/sorafenib therapy, despite inherent sensitivity, but rather reflected the treatment failure observed by lack of growth inhibition. FLT $_{\text {MAX }}$ was increased by $+11 \%(\mathrm{STD} \pm 3 \%, \mathrm{p}=0.06)$ in the TRAIL/ sorafenib treatment group corresponding tumor growth of $302 \%$ $(\mathrm{p}=0.001) . \mathrm{FLT}_{\mathrm{MAX}}$ in the untreated group was $-7 \%(\mathrm{STD} \pm 11 \%$, $\mathrm{p}=0.5)$ with mean tumor growth of $217 \%(\mathrm{p}=0.011)$ in the untreated group.

CALU-6 tumor uptake of FDG was variable following treatment with cisplatin/docetaxel and TRAIL/sorafenib therapy. In therapeutic trials of TRAIL/sorafenib and cisplatin/ docetaxel, overall tumor uptake of $\mathrm{FDG}_{\mathrm{MAX}}$ did decrease posttherapy by as much as $-49 \%$ (Figs. 5 and 6). However, there was variability in this imaging response leading to an overall change in uptake $-8 \%(\mathrm{p}=0.6, \mathrm{STD} \pm 36 \%)$ and $-21 \%(\mathrm{p}=0.3$, $\mathrm{STD} \pm 36 \%$ ) for TRAIL/sorafenib and cisplatin/docetaxel therapy, respectively. In the model of treatment failure, there was a non-statistically significant overall decrease in FDG uptake in the TRAIL/sorafenib and untreated group of $-8 \%$ $(\mathrm{p}=0.4, \mathrm{STD} \pm 39 \%)$ and $-17 \%(\mathrm{p}=0.2, \mathrm{STD} \pm 43 \%)$, respectively.

\section{Discussion}

Cellular proliferation, characteristically high in malignancy, requires a steady intracellular pool of deoxynucleotidetriphosphates (dNTPs). Of these dNTPs, thymidine is specific to DNA synthesis and its metabolism is tightly regulated by cell cycle control mechanisms which source thymidine through two pathways, de novo synthesis, regulated by thymidylate synthase (TS), and the salvage pathway, regulated by thymidine kinase (TK1) (44). TK2, active in the mitochondria, is not cell cycle restricted. FLT, an ${ }^{18} \mathrm{~F}$ analogue of thymidine, exploits the salvage pathway upon which most malignancies are reliant and has a predilection for TK1 rather than TK2 (45). Under wild-type p53 conditions, DNA damage leads to p53-mediated negative regulation of TK 1 followed by halt of cell cycle progression at the $G_{1} / S$ interface which leads to either cell death or senescence $(46,47)$. This cell-cycle regulation of FLT 
accumulation, favorable imaging properties and promising preclinical and clinical trial data have led to intense interest in this radiopharmaceutical as an early indicator of therapeutic response in oncology.

However, the normal relationships between cell cycle progression and TK1 activity are lost in the setting of p53 deficiency. With loss of functioning p53, subsequent DNAdamage results in halting of the cell cycle progression at the $\mathrm{G}_{2} / \mathrm{S}$ checkpoint, rather than $\mathrm{G}_{1} / \mathrm{S}$ checkpoint, allowing accumulation of TK1 in S-phase (47), which potentially limits the utility of FLT to predict therapeutic response in p53-null tumors $(48,49)$. Given that altered p53 function is an extremely common tumor phenotype in general (50) and in NSCLC specifically (1-3) this becomes an important variable to be considered for FLT-PET to successfully enter clinical management.

Our data demonstrate that p53-null NSCLC CALU-6 xeno-grafts treated with cisplatin/docetaxel combination therapy, fail to elicit a predictive drop in FLT accumulation at $72 \mathrm{~h}$ following therapy implementation and do not reflect end-of-therapy growth inhibition and apoptosis relative to untreated controls. Given that imaging is performed before significant therapy induced morphological effects, such as anti-angiogenic activity or tumor shrinkage, are likely to be seen in the xenograft tumors, this discrepancy between imaging therapeutic response prediction and actual tumor response seems most likely either due to a failure of FLT to predict response or suboptimal timing of FLT measurement relative to last delivered therapeutic dose, thus missing the nadir in tumor proliferation.

The failure of FLT to predict therapeutic response in cisplatin/docetaxel therapy may stem from impaired function of p53 in CALU-6. Loss of p53 mediated DNA-damage induced $\mathrm{G}_{1} / \mathrm{S}$ checkpoint activation would allow cell cycle progression into S-phase, despite DNA-damage, allowing accumulation of TK1, and hence FLT, as previously described in other systems (47). Furthermore, the $\mathrm{S} / \mathrm{G}_{2}$ block would halt cell cycle preventing the downregulation and degradation of TK1 seen in $G_{2}$. This hypothesis is supported by the fact that we also did not see suppression of Ki-67 nuclear staining in cisplatin/docetaxel treated tumors, despite the evidence of increased apoptosis and tumor growth inhibition. The Ki-67 nuclear antigen, while not expressed in the resting $\left(\mathrm{G}_{0}\right)$ state, is expressed in the four active stages of the cell cycle, $G_{1}, S, G_{2}$ and $\mathrm{M}$.

Further study is needed with p53 wild-type controls to assess whether cisplatin/docetaxel is able to halt cell cycle progression appropriately in the presence of functional p53. Although a different chemotherapeutic, in vitro exposure to doxorubicin causes p53-null tumor cells to increase TK1 levels 8-36 h following exposure and sustain elevated TK1 levels until 42-48 $\mathrm{h}$ after dosing, allowing expansion of the TTP pool, recovery from chemotherapy induced DNA damage and reentry into mitosis (47). This DNA damage-induced TK1 induction in p53-null cells has been demonstrated to be higher than p53-wild type tumors as a result of $S / G_{2}$ checkpoint activation with $\mathrm{G}_{2}$ arrest (47). It is important to further explore the possible impairment of FLT in the setting of p53-deficiency since this would present a major problem due to the prevalence of the p53-deficient phenotype in clinical practice.
Alternatively, it is possible that due to the weekly dosing schedule of cisplatin/docetaxel, we have missed the maximal impact on cellular proliferation and allowed some degree of recovery from DNA-damage. FLT-PET was performed $72 \mathrm{~h}$ after the 1st weekly dose of cisplatin/docetaxel, both of which have plasma $t_{1 / 2}$ on the order of minutes to hours although cisplatin does have a prolonged protein-bound state. In the clinical setting, therapy with these agents is predicated on fast-acting and sustained DNA-damage allowing for weekly dosing in cancer therapy. However, there is pre-clinical literature suggestive that proliferation may begin to recover in 1-2 days rather than a week. For example, the dose-dependent impact of combination cisplatin/docetaxel therapy on proliferation was found to be maximal at 24-48 $\mathrm{h}$ in the osteosarcoma cell line HOS-8603 in vitro (51) and murine radiation-induced fibrosarcoma (RIF-1) tumors treated with cisplatin result in TK1 protein levels that fall by $24 \mathrm{~h}$ and recover to control levels by $48 \mathrm{~h}$ (13). In support of the 72-h post-treatment window prior to FLT measurement, the p-53 wild-type human squamous cell carcinoma cell line OS-1 demonstrated decreased FLT accumulation following exposure to cisplatin, in vitro, which continued to decrease at $72 \mathrm{~h}$ post-exposure (52). Thus the kinectics of cisplatin/docetaxel and TRAIL/ sorafenib therapy impact on proliferation is an important variable to be considered in the evaluation of FLT as a marker of therapeutic response and will be the study of future work in our laboratory.

Interestingly, while tumor accumulation of FLT declined in TRAIL/sorafenib treated tumors, ultimately correlating with end of therapy marked growth inhibition, decreased tumor angiogenesis, and robust apoptosis, the $\% \mathrm{Ki}-67$ positive nuclear staining of these treated tumors did not decline. We propose two possibilities for this observation. First, sorafenib and TRAIL both have short half-lives with plasma $t_{1 / 2}$ on the order of 3-4 h and $5 \mathrm{~min}$ respectively. As a result in human clinical trials, sorafenib is dosed twice daily and TRAIL is given by continuous i.v. infusion. Therefore, it is possible that TRAIL/sorafenib therapy does transiently hinder proliferation which then recovers as the drug is rapidly eliminated. Our PET examinations were performed on the same day as TRAIL/sorafenib drug dosing whereas tumors were collected for histology approximately $18-24 \mathrm{~h}$ following the last dose of chemotherapy, potentially allowing for recovery of proliferation in viable cells. Secondly, sorafenib is a muti-targeted tyrosine kinase inhibitor with known activity against an increasing range of important targets including RAF/MEK/ERK pathway, PDGFR, c-kit, FLT-3, VEGFR, TGF- $\alpha$ among others. It is possible that the observed drug-induced decreases in FLT accumulation is a result of a block or aberration in thymidine metabolism upstream of TK1 or due to a mechanism totally independent of the DNA salvage pathway and without a direct impact on proliferation.

In conclusion, we propose that FLT accumulation may not be a reliable indicator of treatment success in the setting of a p53-null malignancy. Our results also highlight the need for further characterization of the relationship between the p53 status and predictive ability of FLT-PET and the impact of differences in treatment drug pharmacology on measurement of tumor FLT response in order to maximize the utility of this powerful molecular imaging tool. 


\section{References}

1. Chiba I, Takahashi T, Nau MM, et al: Mutations in the p53 gene are frequent in primary, resected non-small cell lung cancer. Lung Cancer Study Group. Oncogene 5: 1603-1610, 1990.

2. Iggo R, Gatter K, Bartek J, Lane D and Harris AL: Increased expression of mutant forms of p53 oncogene in primary lung cancer. Lancet 335: 675-679, 1990.

3. Bircan A, Bircan S, Kapucuoglu N, et al: Maspin, VEGF and p53 expression in small biopsies of primary advanced lung cancer and relationship with clinicopathologic parameters. Pathol Oncol Res 16: 553-561, 2010.

4. Eisenhauer EA, Therasse P, Bogaerts J, et al: New response evaluation criteria in solid tumours: revised RECIST guideline (version 1.1). Eur J Cancer 45: 228-247, 2009.

5. Kim SJ, Lee JS, Im KC, et al: Kinetic modeling of 3'-deoxy-3'${ }^{18} \mathrm{~F}$-fluorothymidine for quantitative cell proliferation imaging in subcutaneous tumor models in mice. J Nucl Med 49: 2057-2066, 2008.

6. Lawrence J, Vanderhoek M, Barbee D, et al: Use of 3'-deoxy-3'$\left[{ }^{18} \mathrm{~F}\right]$ fluorothymidine $\mathrm{PET} / \mathrm{CT}$ for evaluating response to cytotoxic chemotherapy in dogs with non-Hodgkin's lymphoma. Vet Radiol Ultrasound 50: 660-668, 2009.

7. Debucquoy A, Devos E, Vermaelen P, et al: ${ }^{18} \mathrm{~F}-\mathrm{FLT}$ and ${ }^{18} \mathrm{~F}-\mathrm{FDG}$ PET to measure response to radiotherapy combined with celecoxib in two colorectal xenograft models. Int J Radiat Biol 85: 763-771, 2009.

8. Pantaleo MA, Landuzzi L, Nicoletti G, et al: Advances in preclinical therapeutics development using small animal imaging and molecular analyses: the gastrointestinal stromal tumors model. Clin Exp Med 9: 199-205, 2009.

9. Graf N, Herrmann K, den Hollander J, et al: Imaging proliferation to monitor early response of lymphoma to cytotoxic treatment. Mol Imaging Biol 10: 349-355, 2008.

10. Molthoff CF, Klabbers BM, Berkhof J, et al: Monitoring response to radiotherapy in human squamous cell cancer bearing nude mice: comparison of $2^{\prime}$-deoxy-2'-[ ${ }^{18}$ F]fluoro-D-glucose (FDG) and $3^{\prime}-\left[{ }^{18} \mathrm{~F}\right]$ fluoro-3'-deoxythymidine (FLT). Mol Imaging Biol 9: 340-347, 2007.

11. Buck AK, Kratochwil C, Glatting G, et al: Early assessment of therapy response in malignant lymphoma with the thymidine analogue $\left[{ }^{18} \mathrm{~F}\right] \mathrm{FLT}$. Eur J Nucl Med Mol Imaging 34: 1775-1782, 2007.

12. Chao KS: 3'-deoxy-3'-(18)F-fluorothymidine (FLT) positron emission tomography for early prediction of response to chemoradiotherapy - a clinical application model of esophageal cancer. Semin Oncol 34: S31-S36, 2007.

13. Leyton J, Latigo JR, Perumal M, et al: Early detection of tumor response to chemotherapy by $3^{\prime}$-deoxy $\left.-3^{\prime}-{ }^{18} \mathrm{~F}\right]$ fluorothymidine positron emission tomography: the effect of cisplatin on a fibrosarcoma tumor model in vivo. Cancer Res 65: 4202-4210, 2005.

14. Barthel H, Cleij MC, Collingridge DR, et al: $3^{\prime}$-deoxy- $-3^{\prime}-\left[{ }^{18} \mathrm{~F}\right]$ fluorothymidine as a new marker for monitoring tumor response to antiproliferative therapy in vivo with positron emission tomography. Cancer Res 63: 3791-3798, 2003.

15. Sohn HJ, Yang YJ, Ryu JS, et al: $\left[{ }^{18} \mathrm{~F}\right]$ Fluorothymidine positron emission tomography before and 7 days after gefitinib treatment predicts response in patients with advanced adenocarcinoma of the lung. Clin Cancer Res 14: 7423-7429, 2008

16. Been LB, Hoekstra HJ, Suurmeijer AJ, et al: $\left[{ }^{18} \mathrm{~F}\right]$ FLT-PET and $\left[{ }^{18} \mathrm{~F}\right] \mathrm{FDG}-\mathrm{PET}$ in the evaluation of radiotherapy for laryngeal cancer. Oral Oncol 45: e211-215, 2009.

17. Everitt S, Hicks RJ, Ball D, et al: Imaging cellular proliferation during chemo-radiotherapy: a pilot study of serial ${ }^{18} \mathrm{~F}$-FLT positron emission tomography/computed tomography imaging for non-small-cell lung cancer. Int J Radiat Oncol Biol Phys 75: 1098-1104, 2009

18. Ullrich RT, Zander T, Neumaier B, et al: Early detection of erlotinib treatment response in NSCLC by 3 '-deoxy-3'-[F]fluoro-L-thymidine ([F]FLT) positron emission tomography (PET). PLoS One 3: e3908, 2008.

19. Herrmann K, Wieder HA, Buck AK, et al: Early response assessment using 3 '-deoxy-3'-[ $\left.{ }^{18} \mathrm{~F}\right]$ fluorothymidine-positron emission tomography in high-grade non-Hodgkin's lymphoma. Clin Cancer Res 13: 3552-3558, 2007.

20. Chen W, Delaloye S, Silverman DH, et al: Predicting treatment response of malignant gliomas to bevacizumab and irinotecan by imaging proliferation with $\left[{ }^{18} \mathrm{~F}\right]$ fluorothymidine positron emission tomography: a pilot study. J Clin Oncol 25: 4714-4721, 2007.
21. Herrmann K, Ott K, Buck AK, et al: Imaging gastric cancer with PET and the radiotracers ${ }^{18} \mathrm{~F}$-FLT and ${ }^{18} \mathrm{~F}$-FDG: a comparative analysis. J Nucl Med 48: 1945-1950, 2007.

22. Kenny L, Coombes RC, Vigushin DM, et al: Imaging early changes in proliferation at 1 week post chemotherapy: a pilot study in breast cancer patients with 3 '-deoxy-3'-[ $\left.{ }^{18} \mathrm{~F}\right]$ fluorothymidine positron emission tomography. Eur J Nucl Med Mol Imaging 34: 1339-1347, 2007.

23. Pio BS, Park CK, Pietras R, et al: Usefulness of 3'-[F-18]fluoro3 -deoxythymidine with positron emission tomography in predicting breast cancer response to therapy. Mol Imaging Biol 8: 36-42, 2006

24. Sugiyama M, Sakahara H, Sato K, et al: Evaluation of 3'-deoxy$3^{1}{ }^{18} \mathrm{~F}$-fluorothymidine for monitoring tumor response to radiotherapy and photodynamic therapy in mice. J Nucl Med 45: $1754-1758,2004$

25. Brockenbrough JS, Morihara JK, Hawes SE, et al: Thymidine kinase 1 and thymidine phosphorylase expression in non-smallcell lung carcinoma in relation to angiogenesis and proliferation. J Histochem Cytochem 57: 1087-1097, 2009.

26. Munch-Petersen B, Cloos L, Jensen HK and Tyrsted G: Human thymidine kinase 1 . Regulation in normal and malignant cells. Adv Enzyme Regul 35: 69-89, 1995.

27. Strawn LM, Kabbinavar F, Schwartz DP, et al: Effects of SU101 in combination with cytotoxic agents on the growth of subcutaneous tumor xenografts. Clin Cancer Res 6: 2931-2940, 2000.

28. Miknyoczki SJ,Jones-Bolin S,Pritchard S, et al: Chemopotentiation of temozolomide, irinotecan, and cisplatin activity by CEP-6800, a poly(ADP-ribose) polymerase inhibitor. Mol Cancer Ther 2: 371-382, 2003.

29. Gourdeau H, Leblond L, Hamelin B, et al: Antivascular and antitumor evaluation of 2-amino-4-(3-bromo-4,5-dimethoxyphenyl)-3-cyano-4H-chromenes, a novel series of anticancer agents. Mol Cancer Ther 3: 1375-1384, 2004.

30. Zhang J, Wang X, Lin CJ, Couch FJ and Fei P: Altered expression of FANCL confers mitomycin C sensitivity in Calu-6 lung cancer cells. Cancer Biol Ther 5: 1632-1636, 2006.

31. Teicher BA, Chen V, Shih C, et al: Treatment regimens including the multitargeted antifolate LY231514 in human tumor xenografts. Clin Cancer Res 6: 1016-1023, 2000.

32. Davies BR, Logie A, McKay JS, et al: AZD6244 (ARRY142886), a potent inhibitor of mitogen-activated protein kinase/ extracellular signal-regulated kinase kinase $1 / 2$ kinases: mechanism of action in vivo, pharmacokinetic/pharmacodynamic relationship, and potential for combination in preclinical models. Mol Cancer Ther 6: 2209-2219, 2007.

33. Hurtubise A and Momparler RL: Evaluation of antineoplastic action of 5-aza-2'-deoxycytidine (Dacogen) and docetaxel (Taxotere) on human breast, lung and prostate carcinoma cell lines. Anticancer Drugs 15: 161-167, 2004.

34. Ricci MS, Kim SH, Ogi K, et al: Reduction of TRAIL-induced Mcl-1 and cIAP2 by c-Myc or sorafenib sensitizes resistant human cancer cells to TRAIL-induced death. Cancer Cell 12: 66-80, 2007.

35. Stegehuis JH, De Wilt LH, De Vries EG, et al: TRAIL receptor targeting therapies for non-small cell lung cancer: current status and perspectives. Drug Resist Updat 13: 2-15, 2010.

36. Voortman J, Resende TP, Abou El Hassan MA, Giaccone G and Kruyt FA: TRAIL therapy in non-small cell lung cancer cells: sensitization to death receptor-mediated apoptosis by proteasome inhibitor bortezomib. Mol Cancer Ther 6: 2103-2112, 2007.

37. Spierings DC, De Vries EG, Timens W, et al: Expression of TRAIL and TRAIL death receptors in stage III non-small cell lung cancer tumors. Clin Cancer Res 9: 3397-3405, 2003.

38. Blumenschein GR Jr, Gatzemeier U, Fossella F, et al: Phase II, multicenter, uncontrolled trial of single-agent sorafenib in patients with relapsed or refractory, advanced non-small-cell lung cancer. J Clin Oncol 27: 4274-4280, 2009.

39. Blumenschein G Jr: Sorafenib in lung cancer: clinical developments and future directions. J Thorac Oncol 3: S124-S127, 2008.

40. Kim SH, Kim K, Kwagh JG, et al: Death induction by recombinant native TRAIL and its prevention by a caspase 9 inhibitor in primary human esophageal epithelial cells. J Biol Chem 279: 40044-40052, 2004

41. Dumas J, Hatoum-Mokdad H, Sibley RN, et al: Synthesis and pharmacological characterization of a potent, orally active p38 kinase inhibitor. Bioorg Med Chem Lett 12: 1559-1562, 2002. 
42. Jensen MM, Jorgensen JT, Binderup T and Kjaer A: Tumor volume in subcutaneous mouse xenografts measured by microCT is more accurate and reproducible than determined by ${ }^{18} \mathrm{~F}-\mathrm{FDG}-$ microPET or external caliper. BMC Med Imaging 8: 16, 2008.

43. Ten Tije AJ, Verweij J, Loos WJ and Sparreboom A: Pharmacological effects of formulation vehicles: implications for cancer chemotherapy. Clin Pharmacokinet 42: 665-685, 2003.

44. Hu CM and Chang ZF: Mitotic control of dTTP pool: a necessity or coincidence? J Biomed Sci 14: 491-497, 2007.

45. Bading JR and Shields AF: Imaging of cell proliferation: status and prospects. J Nucl Med 49 (Suppl. 2): S64-S80, 2008.

46. Farnebo M, Bykov VJ and Wiman KG: The p 53 tumor suppressor: a master regulator of diverse cellular processes and therapeutic target in cancer. Biochem Biophys Res Commun 396: 85-89, 2010.

47. Chen YL, Eriksson S and Chang ZF: Regulation and functional contribution of thymidine kinase 1 in repair of DNA damage. J Biol Chem 285: 27327-27335, 2010.
48. Rasey JS, Grierson JR, Wiens LW, Kolb PD and Schwartz JL: Validation of FLT uptake as a measure of thymidine kinase-1 activity in A549 carcinoma cells. J Nucl Med 43: 1210-1217, 2002.

49. Schwartz JL, Tamura Y, Jordan R, Grierson JR and Krohn KA: Effect of p53 activation on cell growth, thymidine kinase-1 activity, and 3'-deoxy-3'fluorothymidine uptake. Nucl Med Biol 31: 419-423, 2004

50. Soussi T and Wiman KG: Shaping genetic alterations in human cancer: the p53 mutation paradigm. Cancer Cell 12: 303-312, 2007.

51. Jin W, Cai L, Niu G and Tao H: Proliferation inhibition effect of docetaxel combined with cisplatin on osteosarcoma cells. Med Oncol 27: 491-494, 2010.

52. Dittmann H, Dohmen BM, Kehlbach R, et al: Early changes in $\left[{ }^{18} \mathrm{~F}\right] \mathrm{FLT}$ uptake after chemotherapy: an experimental study. Eur J Nucl Med Mol Imaging 29: 1462-1469, 2002. 